\title{
The influence of category membership of stimuli on sequential effects in magnitude judgment
}

\author{
PETER PETZOLD and GERT HAUBENSAK \\ Justus Liebig University of Giessen, Giessen, Germany
}

\begin{abstract}
The present study addresses the problem of whether range effects and sequential dependencies are affected by stimulus category membership in the same way. Two sets of squares differing in color were presented to subjects. The subjects were asked to judge them according to size, under two instructions. The separation instruction required them to judge each square solely in relation to its own category. The integration instruction required them to ignore category membership. The results indicated that, under the separation instruction, the range effects, as well as the sequence effects, confined themselves to stimuli of the same category. Under the integration instruction, however, the squares were judged in relation to the range of all the stimuli, and sequential effects were independent of the stimulus category. This parallel trend in the category-specific effects of range and preceding stimuli was found for judgments, as well as for reaction times. The findings are discussed with regard to models of sequential effects.
\end{abstract}

When a series of stimuli varying on one dimension is presented and subjects have to judge the stimuli by category ratings, responses depend not only on the current stimulus, but also on previous stimuli and responses. In numerous studies, a negative correlation between current responses and preceding stimuli, as well as a positive correlation between current responses and preceding responses, has been found (e.g., Jesteadt, Luce, \& Green, 1977; Petzold, 1981; Schifferstein \& Frijters, 1992). These sequential dependencies are affected by several factors, such as the stimulus information available to the observer or the presence or absence of trial-by-trial feedback (e.g., Mori, 1998; Mori \& Ward, 1995; Ward \& Lockhead, 1971). The category membership of the stimuli influences sequential effects as well. If two categories of stimuli are presented in an intermingled series, sequential dependencies are more pronounced when the current stimulus and the preceding one belong to the same category than when they do not (e.g., Luce \& Green, 1978).

To explain sequential effects, some authors have assumed that previous stimulus-response pairs function as a frame of reference for judgments (DeCarlo \& Cross, 1990; Holland \& Lockhead, 1968; Luce \& Green, 1974). According to these authors, a relation between the internal representation $X(t)$ of the stimulus to be judged and the trace $X^{*}(t-1)$ of the preceding stimulus determines

This study was supported by the Deutsche Forschungsgemeinschaft (Ha 936/6). Correspondence should be addressed to P. Petzold, Fachbereich Psychologie, Universität Giessen, Otto-Behaghel-Straße 10, D35394 Giessen, Germany (e-mail: petzold-jena@t-online.de).

Note-This article was accepted by the previous editorial team, headed by Neil Macmillan a relation between the current response $r(t)$ and the preceding one $r(t-1)$. Holland and Lockhead, for example, have proposed a model in which the distance $X(t)-$ $X^{*}(t-1)$ determines the extent to which $r(t)$ differs from $r(t-1)$.

On the other hand, a variety of studies on category judgment have indicated that responses are determined by the total range of the stimulus series. This finding suggests that subjects adjust their reference scales to the endpoints of the subjective range (Parducci, 1965; Petzold, 1982; Witte, 1975). As a consequence, the current stimulus is judged in relation to one or both of the extreme stimuli.

Combining range effects and sequential dependencies, some authors have suggested that both the extreme stimuli and the immediately preceding stimuli may serve as standards for the current stimulus (Baird, Berglund, Berglund, \& Lindberg, 1991; Petzold, 1981). According to this view, both the extremes of the range and the traces of previous stimuli are constituents of frames of reference. Consequently, the influence of the range of stimuli and sequential effects should be governed by the same regularities. In particular, if (1) the stimuli are assigned to disparate categories and (2) category-specific ranges of the attribute under judgment operate as frames of reference, this category specificity should also hold for sequence effects.

The present study was designed to investigate the relation between category-specific ranges and the effect of category membership of successive stimuli on sequential effects in greater detail. By exploring the category specificity of judgments, the aim of the study was to examine some models of sequential effects. In particular, it was to be clarified whether both the extreme stimuli of the range and the traces of preceding stimuli serve as standards for the current stimulus. 
In the following, we will present findings on the category specificity of judgments reported in the literature. Then we will discuss some models of sequential dependencies in relation to category specificity and will present derivations of hypotheses, which were tested in an experiment.

\section{Category-Specific Frames of Reference}

Normally, there is a tendency to judge objects and persons relative to the category they are assigned to. Marks (1988), for example, performed magnitude estimations of loudness for tones of 500 and $2500 \mathrm{~Hz}$, which were presented either in alternating or in random order. In one condition, the sound pressure levels of the $500-\mathrm{Hz}$ tones were high $(50-85 \mathrm{~dB})$, and those of the $2500-\mathrm{Hz}$ tones were low $(35-70 \mathrm{~dB})$. The results indicated that the stimulus category, as defined by the frequency of tones, had a significant effect. The $500-\mathrm{Hz}$ stimuli produced lower loudness judgments than did the $2500-\mathrm{Hz}$ stimuli at equal intensity levels. This effect occurred only if the frequencies fell into different critical bands. Otherwise, the tones were judged independently of their frequencies (Marks $\&$ Warner, 1991). The influence of stimulus categorization on judgments is not at all confined to the auditory modality. Analogous effects have been found for judgments of taste (Rankin \& Marks, 1991) and of length of lines (Armstrong \& Marks, 1997).

The results suggest that frames of reference are category specific. One can distinguish two limiting cases of category specificity. In the first case, each category may form its own frame of reference. Then one obtains psychophysical functions such as those outlined in the left panel of Figure 1. In the opposite case, the category of the stimuli is ignored. Then all the stimuli are judged within the same frame of reference. This leads to psychophysical functions similar to those as shown in the right panel of Figure 1. The curves obtained by Marks (1988) fell somewhere between these limiting cases. These results suggest that two kinds of frames were operative: (1) categoryspecific frames of reference and (2) a frame comprising all the stimuli. Judgments seem to reflect a compromise between these kinds of reference frames.

The extent to which stimulus categories constitute their own frames of reference depends on task variables, such as the instruction. The impact of instructions on judgments has been demonstrated by Parducci, Knobel, and Thomas (1976). They presented subjects with a series of smaller squares and larger circles. When the subjects were instructed to establish separate scales of size for squares and for circles, the frequency distribution of the stimuli of one category did not affect the judgments of stimuli belonging to the other category. However, when the stimuli were presented under the instruction to ignore shape, most subjects were able to integrate both stimulus categories into a single context.

\section{Category-Specific Sequential Effects}

There are relatively few studies concerning the influence of category membership of stimuli on sequential effects. Luce and Green (1978) presented pure tones with frequencies of 1000 or $4000 \mathrm{~Hz}$, whose loudness had to be estimated with the magnitude estimation method. The correlations between successive judgments were lower when the judgments referred to stimuli of different frequencies. In another experiment, Ward (1990) presented tones of two frequencies. The frequencies were selected in such a way that they fell either inside or outside the critical band (Fletcher, 1940) of the other frequency. Those frequencies lying within each other's critical bands were likely to be assigned to the same category. Those lying beyond were likely to belong to different categories. Using regression analysis, Ward (1990) found that sequential effects were stronger when successive stimuli belonged to the same category. In particular, he concluded from the regression coefficients that the contrastive effect of the preceding stimuli was more strongly

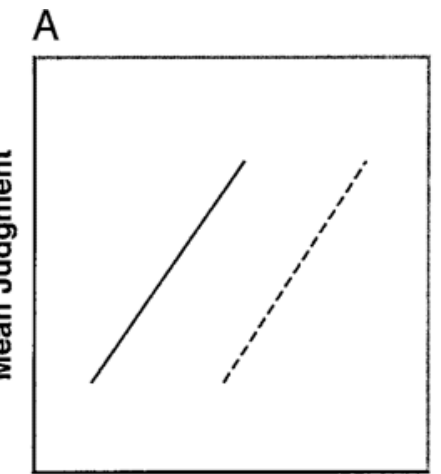

Physical dimension

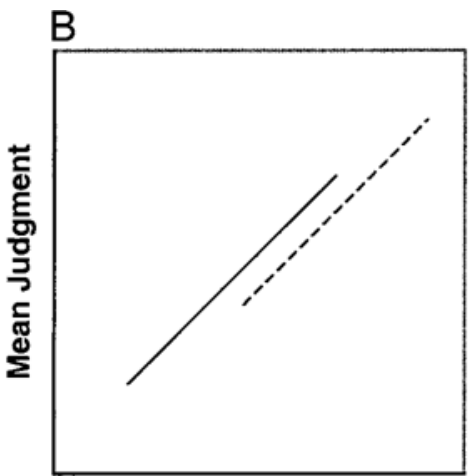

Physical Dimension

Separate frames of reference

$$
\text { Category } 1 \text {.... Category } 2
$$

Figure 1. Mean judgments of stimuli that belong to two categories. Two cases are considered: $A$, in which the stimuli of each category form their own frame of reference, and $B$, in which the category is ignored and the frame of reference is formed by all the stimuli presented. 
affected by the category membership of stimuli than was the assimilative effect of the preceding responses. Similarly, in studies on mixed-modality scaling, Ward (1982, $1985,1986)$ found that assimilation of responses to previous responses occurred within and between modalities, whereas current responses were weakly, if at all, contrasted to previous stimuli of different modality.

The category specificity of frames of reference and of sequential effects has mostly been studied independently up to now. Marks (1993), for example, assumed that sequential dependencies and range effects were caused by two different processes. Whereas sequential assimilation was supposed to result from a relatively late, decisional process, the influence of range was ascribed to categoryspecific sensory adaptation. According to models that assume that both the extremes of range and the preceding stimuli operate as internal standards, however, the effect of categorization on both frames of reference and sequential dependencies is caused by the same process. From this, it follows that range effects and sequential dependencies should exhibit the same regularities. Particularly, when subjects are judging stimuli of different categories separately and provide psychophysical functions like those depicted in the left panel of Figure 1, the magnitude of sequential effects should be higher when the current stimulus and the preceding stimulus belong to the same category. However, when subjects are instructed to ignore the stimulus category and when psychophysical functions like those in the right part of Figure 1 appear, sequence effects should be independent of the category membership of successive stimuli. The present experiment was designed to answer the question of whether the instruction has such an equivalent effect on the range effect and sequential dependencies in category judgment. To formulate more specific hypotheses, we consider some models of sequential dependencies.

\section{Models of Sequential Dependencies}

Several models of sequential dependencies have been suggested, such as the judgment option model (Baird, 1997), the theory of criterion setting (Treisman, 1984), the dynamic response model (DeCarlo \& Cross, 1990), and the consistency model (Haubensak, 1992a). We will not discuss all the models proposed but will focus on two approaches that explicitly comprise the influence of stimulus categorization on sequential effects and the relation between processes underlying the effects of preceding stimuli and responses: (1) models that are based on the relativity of judgments, especially the dynamic judgment theory (DeCarlo \& Cross, 1990) and the multiple standards model (Petzold \& Haubensak, 2001, 2004), and (2) the fuzzy judgment theory (Ward, 1979).

Relativity of judgments. A number of models assume that a relation between the subjective value of the current stimulus $\Psi(t)$ and the trace of the preceding stimulus $\Psi(t-1)$ determines a relation between the current response $r(t)$ and the preceding response $r(t-1)$. For instance, the dynamic response theory of magnitude scal- ing (DeCarlo, 1994; DeCarlo \& Cross, 1990) assumes that judgments are determined by both a long-term frame $\Psi_{0} / r_{0}$ and a short-term frame $\Psi(t-1) / r(t-1)$. Both frames of reference are weighted in the judgment as

$$
r(t)=\left(r_{0} / \Psi_{0}\right)^{1-\lambda} \Psi(t)[r(t-1) / \Psi(t-1)]^{\lambda} v(t),
$$

where $\lambda$ measures the weight of the short-term frame. By taking logarithms and using the power psychophysical function, Equation 1 can be transformed into

$$
\begin{aligned}
\log r(t)= & n \log s(t)-n \lambda \log s(t-1) \\
& +\lambda \log r(t-1)+e(t),
\end{aligned}
$$

where $n$ is the exponent of the power function and $e(t)$ is an error term.

The multiple standards model concerns category judgment and is a generalization of the range model. The range model assumes that stimuli are judged relative to the range they are embedded in (Durlach \& Braida, 1969; Parducci, 1965; Volkmann, 1951; Witte, 1975). This means that judgments are based on a decision continuum, $Z$, given by

$$
Z=\frac{\Psi-\Psi_{\min }}{\Psi_{\max }-\Psi_{\min }},
$$

where $\Psi$ is the subjective value of the stimulus, $\Psi_{\min }$ and $\Psi_{\max }$ are minimum and maximum subjective values of the stimuli defining the endpoints of the range and corresponding to the extreme stimuli. In category judgment tasks, subjects select a response from a relatively small set of integers or word descriptors. There is a lower limit of responses, $r_{\min }$, and an upper limit, $r_{\max }$, both of which are defined by the end categories. According to the range model, the relative deviation of the current response from the lower limit,

$$
\frac{r-r_{\min }}{r_{\max }-r_{\min }},
$$

is determined by the relative deviation of $\Psi$ from $\Psi_{\text {min }}$ given by the value of $Z$.

The range model can be generalized by assuming that not only the extremes of the range, but also previous stimulus-response associations, can serve as referents for the judgment (Haubensak, 1992a; Petzold, 1982). Then two types of standards can be used by the subjects: the extremes of the range as long-term standards and the traces of former stimuli as short-term standards. Whereas the comparatively stable long-term standards are permanently available in memory during an experiment, shortterm standards will be replaced by later ones after some trials (Haubensak, 1992a, 1992b). Which standards are used in a particular trial depends on their position relative to the representation $\Psi$ of the current stimulus: The next lower standard is supposed to serve as a lower standard $\left(\Psi_{1}\right)$, and the next higher standard serves as an upper standard $\left(\Psi_{\mathrm{u}}\right)$. Both the lower and the upper standards can be of the short-term or the long-term type. 
To illustrate this relationship, let us consider the special case in which only the trace of the preceding event, $\Psi(t-1)$ associated with $r(t-1)$, is available in shortterm memory. Then one has to distinguish two subcases. First, if $\Psi_{\text {min }}<\Psi(t-1)<\Psi(t)<\Psi_{\max }, \Psi(t-1)$ will serve as the lower standard and $\Psi_{\max }$ as the upper standard. Second, if $\Psi_{\min }<\Psi(t)<\Psi(t-1)<\Psi_{\max }, \Psi(t-1)$ will be used as the upper standard and $\Psi_{\min }$ as the lower standard.

The choice of a response may be generalized according to the multiple standards model by a process in which the value of

$$
\frac{\Psi-\Psi_{1}}{\Psi_{\mathrm{u}}-\Psi_{1}}
$$

determines the value of

$$
\frac{r-r_{1}}{r_{\mathrm{u}}-r_{1}}
$$

where $r_{1}$ is the response assigned to the stimulus that corresponds to the lower standard $\Psi_{1}$ and $r_{\mathrm{u}}$ is the response assigned to the stimulus that corresponds to the upper standard $\Psi_{\mathrm{u}}$. If, for example, the lower standard $\Psi_{1}$ is the trace of the preceding stimulus, $r_{1}$ is the preceding response.

Concerning the category specificity of judgments, we can derive two hypotheses from the multiple standards model. First, sequential dependencies are assumed to be caused by a process of comparison in which associations between traces of preceding stimuli and preceding responses serve as referents. Consequently, sequential effects concerning stimuli and responses are mediated by the same process. From this it follows that the category membership of successive stimuli should have an equivalent influence on sequential effects concerning stimuli and responses. If some condition-for example, the instruction -increases the absolute value of correlation between current responses and preceding stimuli, the absolute value of the correlation between successive responses should also increase. Second, the multiple standards model assumes that the endpoints of the range and the traces of previous stimuli serve likewise as standards. From this it follows that range effects and sequential dependencies should obey the same regularities when the influence of category membership of stimuli is modified. In particular, if some subjects are instructed to judge stimuli of different categories separately and others are asked to ignore the stimulus category, the influence of category membership should vary in the same direction for the range effect and sequential dependencies. If categoryspecific ranges act as the frame of reference, sequential effects should be stronger for successive stimuli belonging to the same category than for those belonging to different categories. If, however, the overall range is used as the frame of reference, sequential effects should be independent of the category membership of stimuli.

Fuzzy judgment theory. The fuzzy judgment theory assumes that stimuli are internally represented by fuzzy subsets of sensation levels. When choosing a response for category judgment, subjects compare this fuzzy sub- set with prototypes of response categories stored in longterm memory, which are also fuzzy sets. Generally, the result of this match is a set of candidates. To resolve the remaining uncertainty and to select a response, a heuristic procedure is employed that prefers responses similar to the one used previously.

According to this approach, two sequential effects may result. First, due to inhibitory processes in the sensory system, contrastive dependencies on previous stimuli appear. This process is affected by the strength of the interaction between the sensory representations of current stimuli and previous stimuli. The interaction is expected to be higher when the stimuli belong to the same category than when they belong to different categories. Consequently, the contrast with the preceding stimulus should be stronger when successive stimuli share the category. Second, because there is a tendency for the heuristic procedure to select responses close to the preceding response, current responses assimilate to the preceding ones. This is a response-driven process that is independent of the stimuli presented. Thus, the category membership of the stimuli should have no influence on the assimilation toward the preceding response.

\section{Parameters of Linear Regression Equations}

Jesteadt et al. (1977) described sequential dependencies in magnitude estimation by linear regression equations of the form

$$
\begin{aligned}
\log r(t)= & \alpha_{0} \log s(t)+\alpha_{1} \log s(t-1) \\
& +\beta_{1} \log r(t-1)+\delta+\varepsilon(t),
\end{aligned}
$$

where $\delta$ is a constant related to the response scale and $\varepsilon(t)$ is an error term. Although there is no limitation on the parameters $\alpha_{0}, \alpha_{1}$, and $\beta_{1}$ in principle, some models of the judgment process impose restrictions that may serve as a test of the models. For instance, the dynamic judgment theory (DeCarlo \& Cross, 1990) leads to such a restriction. When we compare Equation 2 with the description of sequential dependencies given in Equation 5, we have $\beta_{1}>0$ and $\alpha_{1}<0$. What is most important for testing the model is that we obtain the relation

$$
\alpha_{0} \beta_{1}+\alpha_{1}=0 \text {. }
$$

Similar relations follow from the multiple standards model, which also assumes that both long-term and short-term standards are used in judgments. However, referring to category judgments, the multiple standards model supposes that differences between sensations and standards are decisive in this type of judgment, rather than ratios. Consequently, a regression equation without logarithmic transformations,

$$
\begin{aligned}
r(t)= & a_{0} \Psi(t)+a_{1} \Psi(t-1)+b_{1} r(t-1) \\
& +d+e(t),
\end{aligned}
$$

seems to be appropriate for discussing sequential dependencies in category judgment. To examine whether restrictions of the parameters $a_{0}, a_{1}$, and $b_{1}$ are predicted 
by the multiple standards model, computer simulations were performed in which the number of stimuli, the number of responses, and the discriminability of stimuli were varied. The simulations resulted in $a_{1}<0$ and $b_{1}>$ 0 , and we obtained approximately the relation

$$
a_{0} b_{1}+a_{1}=0
$$

which corresponds to Equation 6.

As DeCarlo and Cross (1990) have shown, a firstorder autoregressive error process is an alternative description of sequential dependencies. Then we have, without the logarithmic transformation,

$$
r(t)=a_{0} \Psi(t)+e(t),
$$

where $e(t)$ is an error term that can be specified by

$$
e(\mathrm{t})=\lambda e(t-1)+u(t),
$$

where $u(t)$ is a random variable with no correlation with its previous values.

It can be shown that if Equation 8 holds true, Equation 7 is equivalent to the first-order autoregressive error process given by Equation 9. Then the autocorrelation parameter $\lambda$ corresponds to the coefficient $b_{1}$ of the preceding response in Equation 7. Describing sequential dependencies as a first-order autoregressive process has the advantage that one parameter, $\lambda$, is sufficient to measure the relative weight of the previous stimulus and the previous response in the subjects' judgments.

Unlike the dynamic judgment theory and the multiple standards model, the fuzzy judgment theory assumes that different processes mediate the influence of the preceding stimuli and the preceding responses. Therefore, no relation between the parameters of the regression equation can be derived.

Events lying two trials back. A number of studies have suggested that stimulus-response events more than one trial back may also affect the response on the current trial. For category judgment, Petzold and Haubensak (2001) have shown that events two trials back may also serve as referents equivalent to the immediately preceding events. This finding suggests generalizing Equation 7 as

$$
\begin{aligned}
r(t)= & a_{0} \Psi(t)+a_{1} \Psi(t-1)+b_{1} r(t-1) \\
& +a_{2} \Psi(t-2)+b_{2} r(t-2)+d+e(t) .
\end{aligned}
$$

Because stimulus-response pairs two trials back are assumed to operate as referents equivalent to the immediately preceding ones, the parameters concerning stimuli and responses of lag 2 should obey the same regularity as the parameters for immediately preceding stimuli and responses. Thus, the multiple standards model predicts

$$
a_{0} b_{2}+a_{2}=0
$$

as an additional relation between parameters.

It can be shown that if the relations in Equations 9 and 11 are true, Equation 10 is equivalent to a second-order autoregressive process

$$
r(t)=a_{0} \Psi(t)+e(t)
$$

with $e(t)=\lambda e(t-1)+\mu e(t-2)+u(t)$. The autocorrelation parameters $\lambda$ and $\mu$ correspond to the coefficients of the previous responses $r(t-1)$ and $r(t-2), b_{1}$ and $b_{2}$ and measure the extent to which the stimulus-response pairs one and two trials back are used as referents.

\section{The influence of category membership}

To study the influence of stimulus categorization quantitatively, the dummy variables, $X$ and $Y$, listed in Table 1, were added to Equation 9.

Then we obtain

$$
\begin{aligned}
r(t)= & a_{0} \Psi(t)+a_{1} \Psi(t-1)+b_{1} r(t-1) \\
& +a_{2} \Psi(t-2)+b_{2} r(t-2)+c X \\
& +u_{1} Y_{1} \Psi(t-1)+v_{1} Y_{1} r(t-1) \\
& +u_{2} Y_{2} \Psi(t-2)+v_{2} Y_{2} r(t-2) \\
& +d+e(t),
\end{aligned}
$$

where $d$ is again a constant depending on the response scale and $e(t)$ is an error term. The coefficient $c$ measures the extent to which category-specific ranges are used in judging stimuli. If judgments are based mainly on category-specific ranges, the value of $c$ should be high. If, however, the range of all the stimuli is applied, $c$ should drop to zero. To understand the meaning of $u_{1}$, $u_{2}, v_{1}$, and $v_{2}$, it may help to rewrite Equation 13 as

$$
\begin{aligned}
r(t)= & a_{0} \Psi(t)+\left(a_{1}+u_{1} Y_{1}\right) \Psi(t-1) \\
& +\left(b_{1}+v_{1} Y_{1}\right) r(t-1) \\
& +\left(a_{2}+u_{2} Y_{2}\right) \Psi(t-2) \\
& +\left(b_{2}+v_{2} Y_{2}\right) r(t-2)+c X+\varepsilon(t) .
\end{aligned}
$$

The coefficient $u_{1}$ describes how strongly the effect of $s(t-1)$ depends on the correspondence between the categories of $s(t)$ and $s(t-1)$. There are two extremes of category specificity. First, if $u_{1}=0$, there is an effect of $s(t-1)$ regardless of the category membership of $s(t)$ and $s(t-1)$. Second, if $u_{1}=-a_{1}$, an effect of $s(t-1)$ exists only when $s(t)$ and $s(t-1)$ belong to the same category. Otherwise, there is no sequential effect at all. The coefficients $u_{2}, v_{1}$, and $v_{2}$ have an equivalent meaning concerning $s(t-2), r(t-1)$, and $r(t-2)$.

Using the coefficients of Equation 13, one can specify three hypotheses concerning the influence of the category membership of stimuli that follow from models of sequential dependencies.

1. According to the dynamic response theory and the multiple standards model, previous stimulus-response pairs serve as referents in judging current stimuli. Therefore, sequential effects concerning preceding stimuli and preceding responses are mediated by the same process. Consequently, they should change concordantly when the influence of category membership is modified, for example, by the instruction. This means that the absolute values of the coefficients $u_{1}$ and $v_{1}$ should change in a 
Table 1

Dummy Variables

\begin{tabular}{lcl}
\hline \multicolumn{1}{c}{ Variable } & Symbol & \multicolumn{1}{c}{ Values } \\
\hline Category of $s(t)$ & $X$ & $\begin{array}{l}\text { 0: category } 2 \\
\text { 1: category 1 }\end{array}$ \\
Correspondence between the categories of $s(t)$ and $s(t-1)$ & $Y_{1}$ & $\begin{array}{l}0: s(t) \text { and } s(t-1) \text { belong to the same category } \\
1: s(t) \text { and } s(t-1) \text { belong to different categories }\end{array}$ \\
Correspondence between the categories of $s(t)$ and $s(t-2)$ & $Y_{2}$ & $\begin{array}{l}0: s(t) \text { and } s(t-2) \text { belong to the same category } \\
1: s(t) \text { and } s(t-2) \text { belong to different categories }\end{array}$ \\
\hline
\end{tabular}

parallel way. This prediction distinguishes the dynamic response theory, as well as the multiple standards model, from the fuzzy judgment model. According to the fuzzy set approach, only the influence of prior stimuli depends on the category membership of those stimuli, whereas the influence of prior responses does not. Consequently, no correlation between $u_{1}$ and $v_{1}$ is to be expected.

2 . The multiple standards model says that the stimuli and responses two trials back may serve as referents, as the immediately preceding stimuli and responses do. From this it follows that any condition that modifies the category specificity of first-order sequential effects also changes the category specificity of second-order sequential effects. Thus, it is predicted that whenever $u_{1}$ and $v_{1}$ are affected, $u_{2}$ and $v_{2}$ are likewise affected.

3. According to the multiple standards model, both the extremes of the range and the preceding stimuli may act as standards. From this it follows that the preceding stimuli should be used in a category-specific way if the category-specific extremes of the range are used this way. Consequently, the values of parameter $c$ and parameters $u_{1}$ and $v_{1}$ should correlate.

\section{Interactions Between the Category \\ Memberships of $s(t-1)$ and $s(t-2)$}

Specific to the multiple standards model is that interactions between the memberships of the categories of the immediately preceding stimulus, $s(t-1)$, and of the stimulus two trials back, $s(t-2)$, can be derived. When $s(t-1)$ and $s(t-2)$ belong to the same category as $s(t)$, both may be used as standards. Then they act concurrently, and the probability that $s(t-1)$ will be used as a standard is lower than when $s(t-2)$ belongs to another category. From this it follows that the correlation between $r(t)$ and $r(t-1)$ should be smaller than it is when only $s(t-1)$ belongs to the same category as $s(t)$. Similarly, the correlation between $r(t)$ and $r(t-2)$ should be smaller when both $s(t)$ and $s(t-1)$ share the same category with $s(t)$ than when only $s(t-2)$ belongs to the same category as $s(t)$. The same conclusions hold for the correlations between the current response and the preceding stimuli.

From the assumptions of the multiple standards model, one can qualify the conditions under which the traces of $s(t-1)$ and $s(t-2)$ may operate concurrently. Prior stimuli will serve as lower standards when they fall below the current stimulus value, and they may be used as upper standards when they are lying above the current stimulus. Because two prior stimuli can act concurrently only if they are lying on the same side of the current stimulus, as in Configuration A in Figure 2, the correlation between $r(t)$ and $r(t-1)$ should be affected by the category membership of $s(t-2)$ only if $s(t-1)$ and $s(t-2)$ are lying on the same side. If they do not, as in Configuration B in Figure 2, the correlation should be invariant with respect to the category of $s(t-2)$.

An analogous conclusion can be drawn for the correlation between $r(t)$ and $r(t-2)$. An effect of the category of stimulus $s(t-1)$ is to be expected only if $s(t-1)$ and $s(t-2)$ are lying on the same side of $s(t)$. Thus, the multiple standards model predicts subtle configural effects of category specificity on sequential dependencies. This would mean that sequential dependencies are affected by the position of the current and previous stimuli on the relevant dimension relative to each other.

The present experiment was designed (1) to test whether both the endpoints of the stimulus range and the preceding stimuli serve internal standards, (2) to examine the relation between the contrast with preceding stimuli and the assimilation toward preceding responses, and (3) to test for the configural effects predicted. Subjects had to judge squares of varying size by category judgment, and different fill styles of squares were used to generate two categories of stimuli. In addition to the judgments, the reaction time (RT) was used as a dependent measure, because the analysis of RTs is a useful tool by which to identify internal standards. That is, the value of RT turned out to be minimal near the position of an internal standard along the dimension under consideration (e.g., Link, 1992).

\section{METHOD}

\section{Stimuli}

The stimulus material consisted of two sets of eight filled and eight empty squares. The squares were presented in an intermingled series. For half the subjects, the filled squares varied from 50 to $71 \mathrm{~mm}$ by steps of $3 \mathrm{~mm}$, and the empty squares varied from 62 to $83 \mathrm{~mm}$; for the other half, the range of the squares was reversed. Thus, each subject saw eight filled and eight empty squares. The overlap between the two stimulus categories (represented by the squares from 62 to $71 \mathrm{~mm}$ ) ensured that the effect of stimulus category on judgments could be studied for four squares of equal size but of different category. The positions of the 16 stimuli on the size dimension are shown in Figure 3.

\section{Instruction}

The subjects' task was to rate the size of squares on a five-step scale as quickly as possible. In Condition S (separation), the subjects were asked to compare each filled square solely with the other filled squares and each empty square solely with the other empty 
squares. In Condition I (integration), the subjects judged the squares under the instruction to ignore the fill style.

\section{Subjects}

Twelve University of Giessen students with various majors took part in the experiment. They were naive with respect to the experimental procedure and received a payment for their participation. They were randomly assigned to two groups that differed in the instruction.

\section{Procedure}

The experiment was run on a commercial computer. After the printed instruction had been read, the squares appeared in random order on the screen. The subjects had to judge each square by pressing the respective key on the computer keyboard. After the keypress, the current square disappeared, and the next square appeared $1 \mathrm{sec}$ later. The session consisted of three blocks lasting $10 \mathrm{~min}$ each. Between the blocks, there was a break of about $5 \mathrm{~min}$.

\section{RESULTS}

\section{Mean Judgments}

Figure 4 represents judgments averaged across all the trials for each category and each instruction separately. One can see that there was a tendency for the stimulus categories to form their own frame of reference under the separation instruction, whereas the impact of categories was weak under the integration instruction. This means that the instructions modified the effect of stimulus categories on judgments in the way expected.

Mean judgments as a function of the width of squares can be approximated as straight lines for both categories and both instructions. A one-way analysis of variance (ANOVA) indicated that there was no significant deviation from the linearity $[F(6,47)<0.20, p>.98]$ for all four cases.

\section{Reaction Time}

We analyzed the RTs as a function of the size of squares. Figure 5 indicates that, under the separation instruction, the RT functions are inversely U-shaped within both stimulus categories. The RT is shorter at the end-

\section{Configuration A}

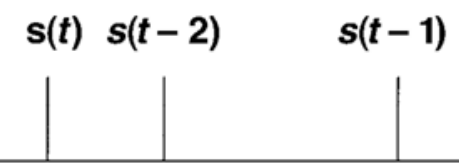

\section{Configuration B}
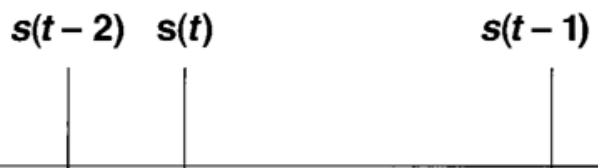

Figure 2. Configurations of $s(t), s(t-1)$, and $s(t-2)$ that differ in the position of $s(t-2)$ relative to $s(t)$ and $s(t-1)$. In Configuration $A, s(t-2)$ lies on the same side of $s(t)$ as $s(t-1)$; in Configuration $B$, it lies on the opposite side. points of the category-specific ranges than in the middle of those ranges. Because of the skewed distribution of RTs, the values were logarithmically transformed before a statistical analysis was performed. For the present data, the mean skewness was 1.86 for the distribution of RTs and 0.30 after the logarithmical transformation. The transformed data were entered into a trend analysis, which confirmed the inversely U-shaped function. The quadratic component was significant for both the lower stimulus category $[F(1,40)=6.34, p=.02]$ and the upper category $[F(1,40)=4.71, p=.03]$.

Under the integration instruction, however, the functions are monotone for both categories. The RT is minimal near the endpoints of the total range. There is no decrease in RTs either at the upper endpoint of the lower stimulus category or at the lower endpoint of the upper one. The quadratic component failed to reach significance for the lower category $[F(1,40)=0.02, p=.91]$, as well as for the upper category $[F(1,40)=1.18, p=$ .28]. Thus, the trends for RT also suggest different uses of frames of reference under the two instructions.

\section{Regression Analyses of Sequential Dependencies}

The analysis of sequential dependencies was based on the coefficients of Equation 13. We confined the analysis up to lag 2, because a former study of size estimation (Petzold \& Haubensak, 2001) indicated that trials further back have no significant influence in category judgment. To estimate the coefficients, the unobservable sensation $\Psi$ must be expressed as a function of physical stimulus magnitude. Because mean judgments as a function of the width of squares turned out to be straight lines, $\Psi$ can be approximated by a linear function,

$$
\Psi=m s+n .
$$

Substituting this function into Equation 13 gives

$$
\begin{aligned}
r(t)= & a_{0}^{*} s(t)+a_{1}^{*} s(t-1)+b_{1} r(t-1) \\
& +a_{2}^{*} s(t-2)+b_{2} r(t-2)+c X \\
& +u_{1}^{*} Y_{1} s(t-1)+v_{1} Y_{1} r(t-1) \\
& +u_{2}^{*} Y_{2} s(t-2)+v_{2} Y_{2} r(t-2)+d^{*} \\
& +e(t),
\end{aligned}
$$

where $a_{0}^{*}=m a_{0}, a_{1}^{*}=m a_{1}, a_{2}^{*}=m a_{2}, u_{1}^{*}=m u_{1}, u_{2}^{*}=$ $m u_{2}$, and $d^{*}=d+n a_{0}+n a_{1}+n a_{2}+n u_{1}+n u_{2}$. The coefficients in Equation 15 were estimated by linear regressions. To ensure that the mean distance between the current stimuli and the preceding stimuli was the same for all cases, only those four stimuli were included in the analysis that fell in the region of overlap between the two stimulus categories (squares from 62 to $71 \mathrm{~mm}$ ). Table 2 summarizes the results for individual data and shows the means for both instructions. We will utilize these results to examine some predictions of the models mentioned above.

Sign of coefficients. The negative sign of $a_{1}^{*}$ means that the previous stimulus exerts a contrast effect on current responses. The coefficient $u_{1}^{*}$ has a positive sign. As 


\section{Stimulus number}

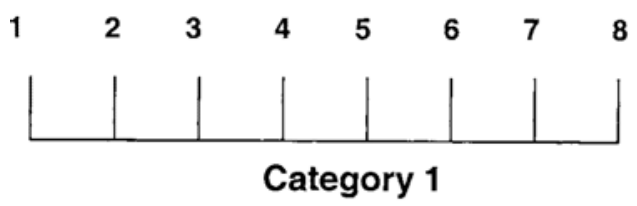

\section{Stimulus number}

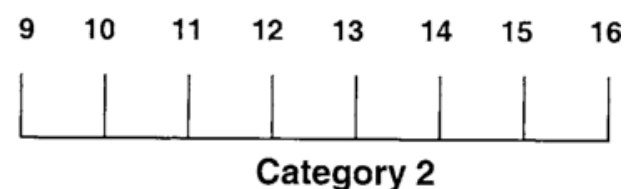

Figure 3. Position of the stimuli used in the experiment on the size dimension.

can be seen from Equation 14, this sign opposite to that of $a_{1}^{*}$ produces a smaller contrast effect when $s(t)$ and $s(t-1)$ belong to different categories $(Y=1)$, as compared with the case of equal categories $(Y=0)$. The positive sign of $b_{1}$ means that the current response is assimilated toward previous responses. Analogously, the opposite, negative sign of $v_{1}$ brings about a reduction of the assimilation effect when $s(t)$ and $s(t-1)$ belong to different categories. The coefficients concerning the event two trials back have corresponding signs.

Relations between coefficients. Corresponding to Equations 8 and 11, the multiple standards model predicts that the terms

$$
\Delta_{1}=a_{0}^{*} b_{1}+a_{1}^{*}
$$

and

$$
\Delta_{2}=a_{0}^{*} b_{2}+a_{2}^{*}
$$

amount to zero. The empirical values of these terms are represented in the last two columns of Table 2. The data confirm the predictions. According to $t$ tests, there were no significant deviations $(p=.05)$ from zero for both terms, either under the separation instruction $[t(6)=$ 3.35 and $t(6)=0.17$ ] or under the integration instruction $[t(6)=0.39$ and $t(6)=0.62]$.

Category specificity of range effect and sequential dependencies. In the foregoing, we hypothesized that the category specificity of sequential dependencies should change whenever the category specificity of the effective range changes. From Table 2, one can see that the value of the coefficient $c^{*}(m=.10)$, which is a measure of the category-specific use of the range, is smaller under the integration instruction than under the separation instruction $(m=.70)$. This difference is significant according to a $t$ test $[t(10)=1.52, p=.001]$. As was expected, the influence of category membership of stimuli on sequential effects shows the same tendency. The absolute values of $u_{1}^{*}[t(10)=3.73], v_{1}^{*}[t(10)=4.88]$, $u_{2}^{*}[t(10)=2.35]$, and $v_{2}^{*}[t(10)=2.30]$ are significantly smaller under the integration instruction than under the
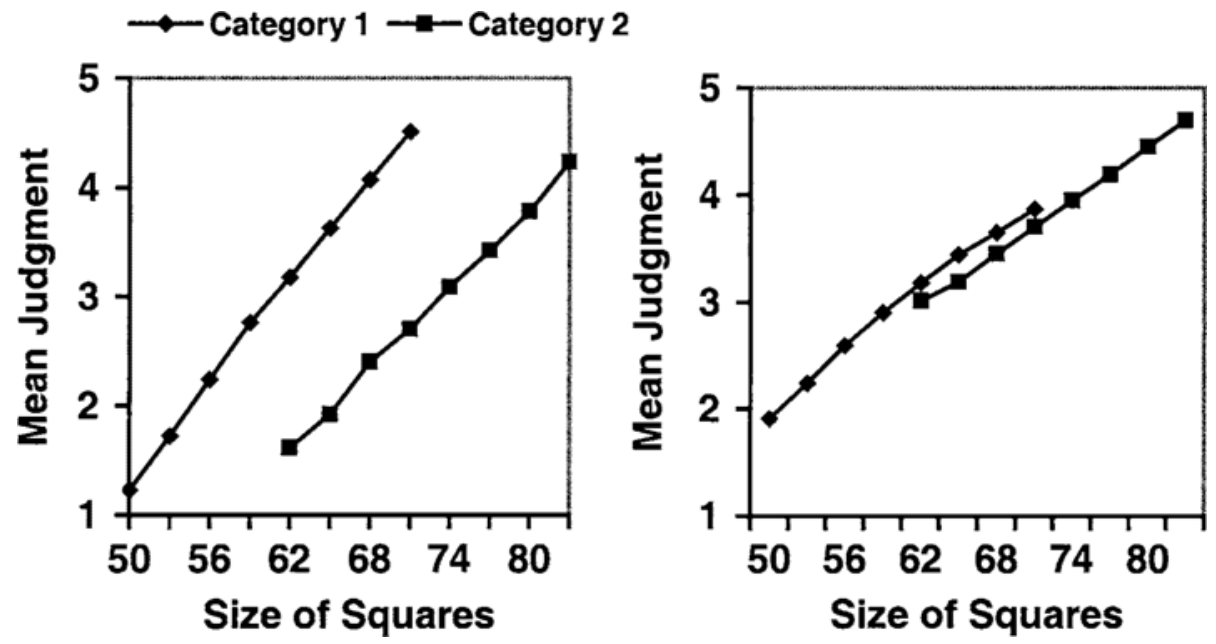

Figure 4. Mean judgment as a function of the size of squares for the two categories of stimuli. Left panel, separation instruction; right panel, integration instruction. 
Table 2

Results of Linear Regression Analyses for Two Instructions.

\begin{tabular}{|c|c|c|c|c|c|c|c|c|c|c|c|c|}
\hline Subject & $a_{0}^{*}$ & $a_{1}^{*}$ & $b_{1}$ & $a_{2}^{*}$ & $b_{2}$ & $c$ & $u_{1}^{*}$ & $v_{1}^{*}$ & $u_{2}^{*}$ & $v_{2}^{*}$ & $\Delta_{1}$ & $\Delta_{2}$ \\
\hline \multicolumn{13}{|c|}{ Separation Instruction } \\
\hline 1 & .493 & -.189 & .514 & -.065 & .111 & .805 & .174 & -.438 & .024 & .027 & .064 & -.010 \\
\hline 2 & .363 & -.217 & .638 & -.079 & .222 & .700 & .219 & -.584 & .081 & -.168 & .015 & .002 \\
\hline 3 & .488 & -.177 & .449 & -.147 & .251 & .589 & .164 & -.412 & .258 & -.510 & .042 & -.025 \\
\hline 4 & .464 & -.199 & .508 & -.053 & .163 & .818 & .165 & -.527 & .074 & -.164 & .037 & .023 \\
\hline 5 & .395 & -.264 & .609 & -.033 & .183 & .394 & .217 & -.588 & .093 & -.203 & -.023 & .039 \\
\hline 6 & .460 & -.210 & .641 & -.089 & .153 & .809 & .200 & -.573 & .023 & -.094 & .085 & -.019 \\
\hline$M$ & .444 & -.209 & .560 & -.078 & .181 & .686 & .190 & -.520 & .092 & -.185 & .037 & .002 \\
\hline \multicolumn{13}{|c|}{ Integration Instruction } \\
\hline 7 & .042 & -.032 & .350 & .019 & .166 & .004 & -.018 & .026 & -.054 & .088 & -.017 & .026 \\
\hline 8 & .316 & -.184 & .544 & -.077 & .163 & .202 & .066 & -.160 & -.015 & .020 & -.012 & -.025 \\
\hline 9 & .304 & -.182 & .594 & -.133 & .218 & .145 & .027 & -.075 & .038 & -.118 & -.001 & -.067 \\
\hline 10 & .186 & -.192 & .722 & .020 & -.048 & -.203 & .203 & -.416 & -.055 & .130 & -.058 & .011 \\
\hline 11 & .316 & -.034 & .392 & -.020 & .108 & .428 & -.099 & .223 & .057 & -.032 & .090 & .014 \\
\hline 12 & .295 & -.141 & .644 & -.062 & .174 & .037 & .011 & -.010 & .015 & -.065 & .049 & -.011 \\
\hline$M$ & .240 & -.128 & .541 & -.042 & .130 & .101 & .032 & -.069 & .002 & .004 & .008 & -.009 \\
\hline
\end{tabular}

separation instruction $(p=.05)$. Thus, the data show a parallel influence of the instruction variable on the range effect and on sequential dependencies.

What is also important is that the modification of the category specificity concerns both the effect of preceding stimuli and the effect of preceding responses. This result is consistent with the hypothesis that the effect of preceding stimuli and responses is mediated by the same process. This hypothesis is further supported by comparing the individual values of $u_{1}^{*}$ and $v_{1}^{*}$ within the instruction groups. We found a significant correlation between $u_{1}^{*}$ and $v_{1}^{*}$ under the separation instruction $[\rho(6)=$ $-.83, p<.05]$, as well as under the integration instruction $[\rho(6)=-.93, p<.05]$; the negative correlations are produced by the opposite signs of $u_{1}^{*}$ and $v_{1}^{*}$. This means that the subjects who showed a strong influence of the correspondence between the categories of $s(t)$ and $s(t-1)$ on the effect of $s(t-1)$ exhibited also a great influence of the category membership of $s(t)$ and $s(t-1)$ on the effect of $r(t-1)$.
Furthermore, the instruction affected the values of $u_{2}^{*}$ and $v_{2}^{*}$ parallel to $u_{1}^{*}$ and $v_{1}^{*}$. This means that the category specificity was modified for stimuli and responses two trials back in the same way as for one trial back. This result supports the assumption that stimulus-response pairs two trials back may also serve as a referent equivalent to the stimulus-response pairs lying one trial back.

To sum up, because the data meet Equations 8 and 11, we can describe mean judgments as the second-order autoregressive process given by Equation 12. Mean values of the coefficients $\lambda$ and $\mu$, which are identical with the coefficients of $r(t-1)$ and $r(t-2)$, are listed in Table 3 for the two instructions and for category membership relations between $s(t), s(t-1)$, and $s(t-2)$. For the separation instruction, the data show that the previous reference frame has no influence on the current response when the category of successive stimuli is different, but it has a large effect when the category is equal. For the integration instruction, the category membership has no effect.

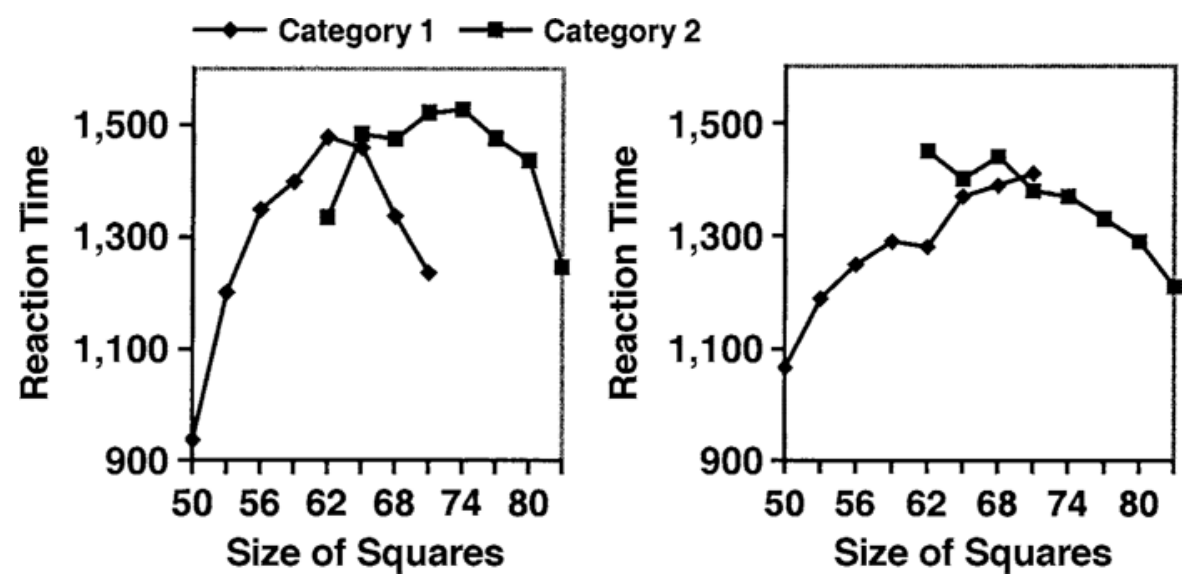

Figure 5. Reaction time as a function of the size of squares for the two categories of stimuli. Left panel, separation instruction; right panel, integration instruction. 


\section{Effect of Stimulus Categories on Sequential Dependencies of RT}

If both the extremes of the range and the traces of preceding stimuli serve as internal standards, equivalent regularities should also show up for range effects and sequential dependencies when RTs are considered. As was shown above, RT is comparatively low for stimuli close to the extremes of the range. This trend has been found for category-specific ranges under the separation instruction, as well as for the overall range under the integration instruction. This pattern should also occur with respect to the distance of the current stimulus to the preceding stimulus. Petzold and Haubensak (2001) have found that RT declines with a decrease of the distance between $s(t)$ and $s(t-1)$. Under the separation instruction, this trend should occur only if successive stimuli belong to the same category. In the case of the integration instruction, however, reducing the distance between successive stimuli should decrease RT independently of the category membership of stimuli.

To test these predictions, we calculated mean RT for (1) each individual, (2) each current stimulus, and (3) several distances between the current and the preceding stimuli separately. The relation between RT and distance $d$ was approximated by a linear function

$$
\mathrm{RT}=a d+b .
$$

The slope $a$ was used as a measure of how strongly the RT depended on the distance between successive stimuli.

Individual values of $a$ and $b$ were estimated by linear regression analyses. Mean slopes averaged over the subjects are represented in Figure 6. For the separation instruction, the slope is higher when successive stimuli belong to the same category than when they belong to different ones. The difference is significant according to a $t$ test $[t(11)=3.09, p=.01]$. For the integration instruction, however, the slope is independent of the category membership of successive stimuli $[t(11)=0.16$, $p=.88]$.

\section{Interaction Between $s(t-1)$ and $s(t-2)$}

As was derived in the introduction, the multiple standards model predicts interactions between the category membership of the immediately preceding stimulus, $s(t-1)$, and the category membership of the stimulus two

Table3

Coefficients $\lambda$ and $\mu$ of the Error Terms $e(t-1)$ and $e(t-2)$ in the Description of Mean Judgments as Second-Order Autoregressive Processes

\begin{tabular}{lcc}
\hline Condition & $\lambda$ & $\mu$ \\
\hline \multicolumn{3}{c}{ Separation Instruction } \\
Category of $s(t), s(t-1)$, and $s(t-2)$ & & \\
Equal & 0.56 & 0.18 \\
Different & 0.02 & 0.00 \\
& & \\
Category of $s(t), s(t-1)$, and $s(t-2)$ & & \\
Equal & 0.54 & 0.13 \\
Different & 0.47 & 0.13 \\
\hline
\end{tabular}

trials back, $s(t-2)$, if the subjects are instructed to isolate the two stimulus categories. When both preceding stimuli belong to the same category as the current stimulus $s(t)$, they should act concurrently as standards. Consequently, the probability that $s(t-1)$ serves as a standard should be lower than when only $s(t-1)$ belongs to the same category as $s(t)$ and $s(t-1)$ does not. This means that sequential effects concerning lag 1 should be weaker when the stimuli of both lag 1 and lag 2 share the category with the current stimulus than when only $s(t-1)$ belongs to the same category as $s(t)$.

To test whether such interactions occur, we analyzed the correlations between the current response and previous responses depending on the category membership of $s(t), s(t-1)$, and $s(t-2)$. In analyzing such interactions, we have to take into account that artifacts may occur. In particular, Gregson (1976) and Haubensak (1992b) have pointed out that judgments averaged over subjects show sequential dependencies even if individual data do not, because some individuals tend to judge the stimuli as all high and others tend to judge them as all low. Such pseudosequential effects may occur even in the individual data averaged over an experiment if the responses systematically drift during the course of the experiment. There are several methods by which to avoid such artifacts. Schifferstein and Kuiper (1997), for example, used standardized responses to correct for differences in scale usage. This brought the responses down to a mean of 0 and a standard deviation of 1 . By following this procedure, we determined mean $m$ and standard deviation $\sigma$ of the responses $r$ and calculated standardized responses $r_{s}=$ $(r-m) / \sigma$ for each subject and each part of a session separately. These standardized responses were then used to analyze interaction in sequential dependencies.

According to the hypothesis, the correlation between $r_{s}(t)$ and $r_{s}(t-1)$, as well as the correlation between $r_{s}(t)$ and $s(t-1)$, should be lower if $s(t-1)$ and $s(t-2)$ belong to the same category as $s(t)$ than when only $s(t-1)$ shares the category with $s(t)$. To test this prediction, we calculated individual correlations for all combinations of category membership for $s(t), s(t-1)$, and $s(t-2)$ under the separation instruction. To eliminate a possible distance effect, only the stimuli in the region of overlap between the two stimulus categories were included in the analysis. This procedure ensured that the mean distance between the values of $s(t)$ and $s(t-1)$, as well as the distance between $s(t)$ and $s(t-2)$, was the same for all combinations of categories. First, we compared correlations between successive responses (1) when both $s(t-2)$ and $s(t-1)$ belonged to the same category as $s(t)$ and (2) when only $s(t-1)$ belonged to the same category. In the first case, the mean value averaged over subjects and the category of current stimuli was .38; in the latter case, it was .60. According to a $t$ test of the $z$-transformed correlations, this difference is significant $[t(11)=3.88, p<$ $.05]$. We obtained similar results for the correlation between current responses and the preceding stimulinamely, mean $\rho\left[r_{\mathrm{s}}(t), s(t-1)\right]=-.38$ when $s(t-1)$ and $s(t-2)$ belonged to the same category as $s(t)$, and 


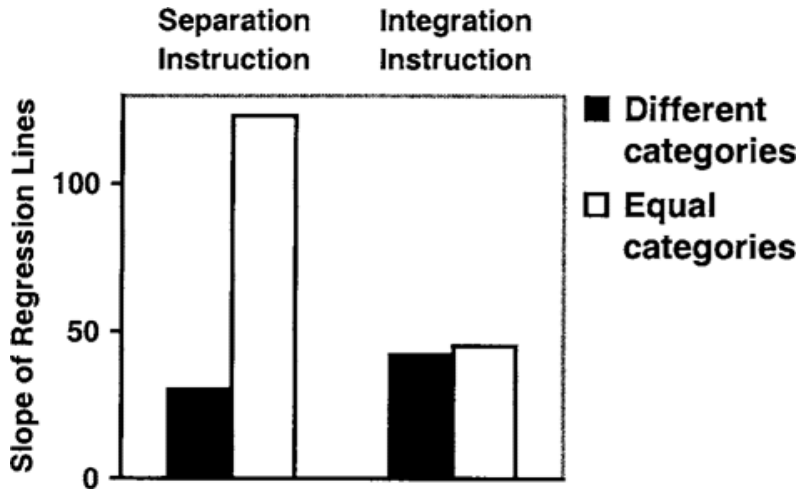

Figure 6. Slopes of the linear regression equation $\mathrm{RT}=\boldsymbol{a d}+\boldsymbol{b}$, where $d$ is the distance between the current stimulus and the preceding stimulus.

mean $\rho\left[r_{\mathrm{s}}(t), s(t-1)\right]=-.54$ when only $s(t-1)$ shared the category with $s(t)$. The difference between the $z$ transformed correlations is also significant $[t(11)=$ $2.57, p<.05]$.

Configural effects. The multiple standards model predicts special configural effects with regard to the positions of $s(t-1)$ and $s(t-2)$ relative to $s(t)$. As was derived in the introduction, a first prediction is that the correlation between $r(t)$ and $r(t-1)$ should be affected by the category membership of $s(t-2)$ only if both $s(t-1)$ and $s(t-2)$ fall on the same side of $s(t)$. Otherwise, the correlation should not depend on the category of $s(t-2)$. To test this prediction, all those pairs of configurations were selected that met the following conditions: First, the position of $s(t), s(t-1)$, and $s(t-2)$ along the size dimension was held constant, whereas the membership of $s(t-2)$ to categories was changed within a pair of configurations. Second, those current stimuli are chosen which are preceded by an equal number of smaller and larger stimuli. These pairs are listed in Table 4. The numbering of stimuli corresponds to that in Figure 2. As an example, consider the pair of configurations represented on the first two lines of Table 4 . In both configurations, $s(t)$ and $s(t-1)$ belong to category $1, s(t)$ has the value 4 or 5 , and $s(t-1)$ has the value 1,2 , or 3 . The position of $s(t-2)$ on the size dimension is the same in both configurations; it lies on the opposite side of $s(t)$ as compared with $s(t-1)$. The category of $s(t-2)$ is varied: $s(t-2)$ belongs to category 1 in the first configuration $[s(t-2)=6,7$, or 8], whereas it belongs to category 2 in the second configuration $[s(t-2)=$ 10,11 , or 12]. Because $s(t-1)$ and $s(t-2)$ does not fall on the same side of $s(t)$, the category membership of $s(t-2)$ should not affect the correlation between $r(t)$ and $r(t-1)$. If, however, $s(t-1)$ and $s(t-2)$ fall on the same side of $s(t)$ on the size dimension, as in the configurations of lines 5 and 6 , the correlation should be smaller if $s(t-1)$ and $s(t-2)$ belong to the same category than if they belong to different categories.

To test the hypothesis that the category membership of $s(t-2)$ affects $\rho\left[r_{\mathrm{s}}(t), r_{\mathrm{s}}(t-1)\right]$ only if $s(t-1)$ and $s(t-2)$ are on the same side of $s(t)$, we entered the $z$ transformed correlations represented in Table 4 into a 2 [category membership of $s(t-1)$ and $s(t-2)$ : equal or different] $\times 2$ [position of $s(t-1)$ and $s(t-2)$ relative to $s(t)$ : equal or different sides] ANOVA. The interaction between category membership and position was significant $[F(1,2)=15.8, p<.05]$.

An equivalent result was obtained for the correlation between $r_{\mathrm{s}}(t)$ and $r_{\mathrm{s}}(t-2)$. The configurations presented in Table 5 were selected according to principles corresponding to those for configurations shown in Table 4. The correlation coefficients suggest that category membership of $s(t-1)$ has an effect only if $s(t-1)$ and $s(t-2)$ are on the same side of $s(t)$. Again, we performed an ANOVA with the $z$-transformed correlation coefficients. This time, the interaction between category membership of $s(t-1)$ and $s(t-2)$ and the positions of $s(t-1)$ and $s(t-2)$ was only marginally significant $[F(1,2)=6.73, p=.06]$.

\section{DISCUSSION}

\section{Category Specificity of Judgments}

The results of the present experiment showed that the instruction modified the judgments. When the subjects

Table 4

Correlations Between Successive Responses for Configurations of $s(t), s(t-1)$, and $s(t-2)$ Differing With Respect to the Position of the Preceding Stimuli

Relative to the Current Stimulus and in the Correspondence of the Category Membership of $s(t-1)$ and $s(t-2)$

\begin{tabular}{crrrc}
\hline Category of $s(t-1)$ and $s(t-2)$ & $s(t)$ & $s(t-1)$ & $s(t-2)$ & $\rho\left[r_{s}(t), r_{\mathrm{s}}(t-1)\right]$ \\
\hline & $s(t-1)$ and $s(t-2)$ on Different Sides of $s(t)$ & \\
Equal & $4 \ldots 5$ & $1 \ldots 3$ & $6 \ldots 8$ & .64 \\
Different & $4 \ldots 5$ & $1 \ldots 3$ & $10 \ldots 12$ & .61 \\
Equal & $12 \ldots 13$ & $14 \ldots 16$ & $9 \ldots 11$ & .55 \\
Different & $12 \ldots 13$ & $14 \ldots 16$ & $5 \ldots 7$ & .50 \\
& $s(t-1)$ and $s(t-2)$ on the Same Side of $s(t)$ & \\
Equal & $4 \ldots 5$ & $6 \ldots 8$ & $6 \ldots 8$ & .34 \\
Different & $4 \ldots 5$ & $6 \ldots 8$ & $10 \ldots 12$ & .60 \\
Equal & $12 \ldots 13$ & $9 \ldots 11$ & $9 \ldots 11$ & .38 \\
Different & $12 \ldots 13$ & $9 \ldots 11$ & $5 \ldots 7$ & .62 \\
\hline
\end{tabular}


were instructed to compare filled squares only with other filled squares and empty squares only with other empty squares (separation instruction), the stimuli were rated relative to their own category. When, however, the subjects were asked to ignore the fill style (integration instruction), the stimuli were judged relative to the range of all squares. These findings indicate, once more, (1) the flexibility in category building and (2) the importance of position within the relevant range. Under the separation instruction, there were filled and empty squares that were judged to be equal although their sizes were different. Moreover, filled and empty squares of the same size were judged to be different. These findings indicate that it is not the absolute size that is important for the judgment of stimuli, but their position within the relevant range. Under the separation instruction, empty and filled squares were judged to be equal if they shared the position within their own range.

\section{Extremes of the Range and Preceding Stimuli}

The findings of the present experiment indicate that the instruction affects the category specificity of both mean judgments and sequential dependencies in the same way. If subjects are instructed to compare stimuli only with those of the same category, there is a tendency to rate the stimuli relative to the category-specific range. Concomitantly, sequential effects are stronger when successive stimuli belong to the same category than when they do not. If, however, subjects are instructed to ignore the category of stimuli, they use the total range of stimuli as a frame of reference, and concomitantly, sequential effects are no longer dependent on the category membership of successive stimuli. This equivalence of category specificity holds for both the immediately preceding presentation and the presentation two trials back.

Such a parallel trend for category specificity with respect to the extremes of the range and the preceding stimuli holds also for RTs. RT decreases with increasing distance of the current stimulus from the extremes of the range and with increasing distance from the preceding stimulus as well. Under the separation instruction, the distance from category-specific extremes and from stimuli of the same category matters. Under the integration instruction, however, category membership of stimuli has no substantial effect on RT. All these findings support the claim that the endpoints of the stimulus range and the traces of preceding stimuli have an equivalent function in the judgment process.

\section{Relation Between Sequential Effects Concerning Preceding Stimuli and Preceding Responses}

Models such as the dynamic judgment model (DeCarlo \& Cross, 1990), the consistency model (Haubensak, 1992a) and the multiple standards model (Petzold \& Haubensak, 2001) assume that the effects of the preceding stimuli and the preceding responses are mediated by the same process. This notion is corroborated by the results of linear regression analyses in which the coefficients of previous stimuli and responses meet the relations in Equations 8 and 11, derived from models that assume that previous stimulus-response pairs operate as referents.

Additional support for this assumption is provided by the finding that the category membership of successive stimuli affects the effect of preceding stimuli and preceding responses in an equivalent manner. That is, the effect of both the preceding stimuli and the preceding responses was smaller when successive stimuli belonged to different categories than when they belonged to the same category.

A further analogy between the influence of preceding stimuli and preceding responses is provided by the interaction of events one and two trials back. As was shown above, the correlation between the current response and the immediately preceding stimulus, as well as the correlation between successive responses, was greater when only the immediately preceding stimulus had the category in common with the current stimulus than when the stimulus two trials back also belonged to the same category as the current stimulus. This finding supports the assumption that the same process underlies sequential effects concerning preceding stimuli and preceding responses.

Table 5

Correlations Between the Current Response and the Response Two Trials Back for Configurations of $s(t), s(t-1)$, and $s(t-2)$ Differing With Respect to the Position of the Preceding Stimuli Relative to the Current Stimulus and in the Correspondence of the Category Membership of $s(t-1)$ and $s(t-2)$

\begin{tabular}{crrrr}
\hline Category of $s(t-1)$ and $s(t-2)$ & $s(t)$ & $s(t-1)$ & $s(t-2)$ & $\rho\left[r_{\mathrm{s}}(t), r_{\mathrm{s}}(t-2)\right]$ \\
\hline \multicolumn{4}{c}{$s(t-1)$ and $s(t-2)$ on Different Sides of $s(t)$} \\
Equal & $4 \ldots 5$ & $6 \ldots 8$ & $1 \ldots 3$ & .32 \\
Different & $4 \ldots 5$ & $10 \ldots 12$ & $1 \ldots 3$ & .27 \\
Equal & $12 \ldots 13$ & $9 \ldots 11$ & $14 \ldots 16$ & .15 \\
Different & $12 \ldots 13$ & $5 \ldots 7$ & $14 \ldots 16$ & .11 \\
& $s(t-1)$ and $s(t-2)$ on the Same Side of $s(t)$ & \\
Equal & $4 \ldots 5$ & $6 \ldots 8$ & $6 \ldots 8$ & .05 \\
Different & $4 \ldots 5$ & $10 \ldots 12$ & $6 \ldots 8$ & .33 \\
Equal & $12 \ldots 13$ & $9 \ldots 11$ & $9 \ldots 11$ & .07 \\
Different & $12 \ldots 13$ & $5 \ldots 7$ & $9 \ldots 11$ & .31
\end{tabular}




\section{Alternative Psychophysical Tasks}

To study sequential dependencies, we used category ratings in the present experiment. Typical effects such as contrast with the preceding stimuli and assimilation toward the preceding response appear not only in category judgment, but also in other psychophysical tasks, such as magnitude estimation (e.g., DeCarlo \& Cross, 1990; Jesteadt et al., 1977), cross-modality matching (Ward, 1979), magnitude production (DeCarlo, 2003), and twostimulus identification with confidence judgment (McNicol, 1980). Also, the category specificity of sequential dependencies has been found in other tasks, such as magnitude estimation (Luce \& Green, 1978) and mixedmodality matching (Ward 1982, 1985, 1986).

These findings suggest that previous stimulus-response associations generally serve as referents in judgment processes. However, in which manner preceding stimuli and responses are involved seems to be different for particular tasks. Petzold and Haubensak (2001) found that stimulus-response pairs two trials back operated in a way equivalent to the immediately preceding ones only for category judgment, but not for magnitude estimation. This result suggests that two prior stimuli can be included in the judgment process in the case of category judgment, whereas the relation to one prior stimulus seems to be decisive for magnitude estimation.

In the present experiment, we found interactions between stimuli one and two trials back with respect to membership in stimulus categories. Moreover, configural effects indicated that the position of $s(t), s(t-1)$, and $s(t-2)$ relative to each other is important for the influence of category membership. In view of the results found by Petzold and Haubensak (2001), we can speculate that these findings are specific to category judgment. Whether this assumption can be confirmed or whether similar interactions also occur in other psychophysical tasks remains to be clarified.

\section{Models of Sequential Dependencies}

Two types of models for the influence of stimulus categorization on sequential dependencies were compared in the introduction: (1) relativity of judgment - in particular, the dynamic judgment theory (DeCarlo \& Cross, 1990) and the multiple standards model (Petzold \& Haubensak, 2001) - and (2) the fuzzy judgment theory (Ward, 1979).

According to relativity-of-judgment models, preceding stimulus-response pairs are used as referents in the judgment process, producing both contrast with the preceding stimuli and assimilation toward the preceding responses. The fuzzy judgment theory assumes that contrast with the preceding stimulus is caused by a sensory process and assimilation toward the preceding response is mediated by a heuristic that selects the overt response from a set of acceptable responses.

The relativity-of-judgment models are supported by the close relationship between sequential effects concerning preceding stimuli and preceding responses. Especially, the multiple standards model for category judgment is corroborated by the finding that the immediately preceding event and the event two trials back show the same regularities of sequential dependencies. They show an analogous relation between the parameters in the linear regression equations, and the influence of category membership of stimuli is the same. A further argument for the multiple standards model is provided by the configural effects shown in the present experiment. The category membership of the stimulus two trials back, $s(t-2)$, affects the correlation between successive responses only if it is positioned on the same side of the current stimulus, $s(t)$, as the immediately preceding stimulus $s(t-1)$. When $s(t-2)$ and $s(t-1)$ lie on different sides of $s(t)$, the category membership of $s(t-2)$ has no influence on the correlations. This finding is predicted by the multiple standards model, which claims that two standards are operative in each trial; a lower standard located below the current stimulus and an upper standard located above the current stimulus. If $s(t-1)$ and $s(t-2)$ lie on the same side of $s(t)$, only one of them can act as a standard, and consequently, the two stimuli operate concurrently and the correlation is low. If they fall on different sides, however, they may both operate as standards, and consequently, the correlations are higher.

Ward (1990), in contrast, found in his study on loudness scaling that the contrastive effect of the preceding stimuli was affected by the category membership of stimuli more strongly than was the assimilative effect of the preceding response. He interpreted this finding as indicating different processes for the effect of preceding stimuli and preceding responses. To reconcile the findings of Ward and the results of the present experiment, one might hypothesize that although the reference to preceding stimulus-response pairs is the essential process underlying sequential effects, an additional responsedriven component produces additional sequential dependencies that are independent of the category membership of the stimuli. This additional component could be a guessing strategy by which a response is selected from a subset of acceptable responses, with the tendency to minimize the difference between the values of the preceding response and the current one (Petzold, 1981; Ward \& Lockhead, 1971). This component corresponds to the heuristic included in the fuzzy judgment theory (Ward, 1979), which takes into account prior responses only. According to this model, sequential effects are produced by the superposition of two processes: (1) using preceding stimulus-response pairs as referents and (2) selecting a response from a subset of alternatives by guessing. Another process that might be at work, in addition to the comparison process, could be the stimulus context effect proposed by DeCarlo and Cross (1990). According to this view, the perception of the current stimulus is affected by the stimulus context in which the preceding stimulus is an essential constituent. If we assume that the influence of the context is contrastive and category specific, the results found by Ward (1990) are consistent with the stimulus context approach. Further research is needed to clarify whether a two-component model can explain all the findings on sequential dependencies and what the nature of the additional component is. 


\section{REFERENCES}

Armstrong, L., \& Marks, L. E. (1997). Differential effects of stimulus context on perceived length: Implications for the horizontal-vertical illusion. Perception \& Psychophysics, 59, 1200-1213.

BAIRD, J. C. (1997). Sensation and judgment. Mahwah, NJ: Erlbaum. Baird, J. C., Berglund, B., Berglund, U., \& Lindberg, S. (1991). Stimulus sequence and the exponent of the power function for loudness. Perceptual \& Motor Skills, 73, 3-17.

DeCARLO, L. T. (1994). A dynamic theory of proportional judgment: Context and judgment of length, heaviness, and roughness. Journal of Experimental Psychology: Human Perception \& Performance, 20, 372-381.

DeCARLO, L. T. (2003). An application of the dynamic model of judgment to magnitude production. Perception \& Psychophysics, 65, 152-162.

DeCarlo, L. T., \& Cross, D. V. (1990). Sequential effects in magnitude scaling: Models and theory. Journal of Experimental Psychology: General, 119, 375-396.

Durlach, N. I., \& BraidA, L. D. (1969). Intensity perception: Preliminary theory of intensity resolution. Journal of the Acoustical Society of America, 46, 372-383.

FLETCHER, H. (1940). Auditory patterns. Reviews of Modern Physics, 12, 47-65.

GREGSON, R. A. M. (1976). Psychophysical discontinuity and pseudosequence effects. Acta Psychologica, 40, 431-451.

HAUBENSAK, G. (1992a). The consistency model: A process model for absolute judgment. Journal of Experimental Psychology: Human Perception \& Performance, 18, 303-309.

HAUBENSAK, G. (1992b). Sequenzeffekte in absoluten Urteilen? Kritisches zur Methode. Zeitschrift für Experimentelle und Angewandte Psychologie, 39, 101-113.

Holland, H. K., \& LoCKHEAd, G. R. (1968). Sequential effects in absolute judgments of loudness. Perception \& Psychophysics, 3, 409414.

Jesteadt, W., Luce, R. D., \& Green, D. M. (1977). Sequential effects in judgments of loudness. Journal of Experimental Psychology: Human Perception \& Performance, 3, 92-104.

LINK, S. W. (1992). The wave theory of difference and similarity. Hillsdale, NJ: Erlbaum.

LuCE, R. D., \& GREEN, D. M. (1974). The response ratio hypothesis for magnitude estimation. Journal of Mathematical Psychology, 11, 114.

Luce, R. D., \& Green, D. M. (1978). Two tests of a neural attention hypothesis for auditory psychophysics. Perception \& Psychophysics, 23, 363-371.

MARKS, L. E. (1988). Magnitude estimation and sensory matching. Perception \& Psychophysics, 43, 511-526.

MARKS, L. E. (1993). Contextual processing of multidimensional and unidimensional auditory stimuli. Journal of Experimental Psychology: Human Perception \& Performance, 19, 227-249.

MARKs, L. E., \& WARNER, E. (1991). Slippery context effect and critical bands. Journal of Experimental Psychology: Human Perception \& Performance, 17, 986-996.

MCNICOL, D. (1980). Multiple regression analyses of sequential effects in loudness judgment. In R. S. Nickerson (Ed.), Attention and performance VIII (pp. 179-196). Hillsdale, NJ: Erlbaum.

MoRI, S. (1998). Effects of stimulus information and number of stimuli on sequential dependencies in absolute identification. Canadian Journal of Experimental Psychology, 52, 72-83.

MORI, S., \& WARD, L. M. (1995). Pure feedback effects in absolute identification. Perception \& Psychophysics, 57, 1065-1079.
PARducci, A. (1965). Category judgment: A range-frequency model. Psychological Review, 72, 407-418.

PARDUCCI, A., KNOBEL, S., \& Thomas, C. (1976). Independent contexts for category ratings: A range-frequency analysis. Perception \& Psychophysics, 20, 360-366.

Petzold, P. (1981). Distance effects on sequential dependencies. Journal of Experimental Psychology: Human Perception \& Performance, 7, 1371-1385.

PeTZOLD, P. (1982). The edge effect of discriminability in categorical judgments. In H.-G. Geissler \& P. Petzold (Eds.), Psychophysical judgment and the process of perception (pp. 222-232). Amsterdam: North-Holland.

Petzold, P., \& Haubensak, G. (2001). Higher order sequential effects in psychophysical judgments. Perception \& Psychophysics, 63, $969-$ 978.

Petzold, P., \& Haubensak, G. (2004). Short-term and long-term frames of reference: A multiple standards model. In C. Kaernbach, E. Schröger, \& H. Müller (Eds.), Psychophysics beyond sensation: Laws and invariants of human cognition (pp. 45-68). Mahwah, NJ: Erlbaum.

RANKIN, K. M., \& MARKs, L. E. (1991). Differential context effects in taste perception. Chemical Senses, 16, 617-629.

Schifferstein, H. N. J., \& Frijters, J. E. R. (1992). Contextual and sequential effects on judgments of sweetness intensity. Perception \& Psychophysics, 52, 243-255.

Schifferstein, H. N. J., \& KuIPER, W. E. (1997). Sequence effects in hedonic judgments of taste stimuli. Perception \& Psychophysics, $\mathbf{5 9}_{2}$ 900-912.

TrEISMAN, M. (1984). A theory of criterion setting: An alternative to the attention band and response ratio hypotheses in magnitude estimation and cross-modality matching. Journal of Experimental Psychology: General, 113, 443-463.

VOLKMANN, J. (1951). Scales of judgment and their implications for social psychology. In J. H. Rohrer \& M. Sherif (Eds.), Social psychology at the crossroads (pp. 279-294). New York: Harper \& Row.

WARD, L. M. (1979). Stimulus information and sequential dependencies in magnitude estimation and cross-modality matching. Journal of Experimental Psychology: Human Perception \& Performance, $\mathbf{5}_{2}$ 444-459.

Ward, L. M. (1982). Mixed-modality psychophysical scaling: Sequential dependencies and other properties. Perception \& Psychophysics, 31, 53-62.

WARD, L. M. (1985). Mixed-modality psychophysical scaling: Interand intramodality sequential dependencies as a function of lag. Perception \& Psychophysics, 38, 512-522.

WARD, L. M. (1986). Mixed-modality psychophysical scaling: Double cross-modality matching for "difficult" continua. Perception \& Psychophysics, 39, 407-417.

WARD, L. M. (1990). Critical bands and mixed-frequency scaling: Sequential dependencies, equal-loudness contours, and power function exponents. Perception \& Psychophysics, 47, 551-562.

Ward, L. M., \& LocKHEAD, G. R. (1971). Response system processes in absolute judgment. Perception \& Psychophysics, 9, 75-78.

Witte, W. (1975). Zum Gestalt- und Systemcharakter psychischer Bezugssysteme. In S. Ertel (Ed.), Gestalttheorie in der modernen Psychologie (pp. 171-182). Darmstadt: Steinkopf.

(Manuscript received September 20, 2001; revision accepted for publication September 25, 2003.) 